\title{
Clinical Profile of Narcolepsy in Children in India
}

\section{Arun Grace Roy ${ }^{*}$, Siby Gopinatha, Suresh Kumara, Dinesh Singh ${ }^{a}$, Sheela Nampoodirib, Vinayan K Pa, Anand Kumara. Mali A. Einenc, Emmanuel Mignotc}

a Department of Neurology, Amrita Institute of Medical Sciences, Kochi 682041, Kerala

b Department of Pediatric Genetics, Amrita Institute of Medical Sciences, Kochi 682041, Kerala, India

c Stanford University Center For Narcolepsy, 3165 Porter Drive, Palo Alto, CA 94304, USA

DOI No:10.5958/0974-0155.2015.00011.X

Indian J Sleep Med 2015; 10.2, 74-78

\begin{abstract}
Objective/Background: Narcolepsy is a chronic disorder characterized by excessive daytime somnolence (EDS), sleep paralysis, hypnagogic or hypnopompic hallucinations and cataplexy. Symptoms of narcolepsy typically start in childhood and young adult and clinical picture can be variable. In this article, the clinical picture, and investigations of 19 children with diagnosis of narcolepsy are described. The objective of this study is to describe the clinical profile, polysomnography (PSG) and multiple sleep latency test (MSLT) features and investigations in 19 children (<18years).

Materials and Methods: This is a descriptive retrospective study reporting on the clinical and biological features of children diagnosed with narcolepsy in the sleep center. All children underwent overnight PSG followed by MSLT. HLA analysis and CSF hypocretin assay were part of protocol but HLA analysis was done only in 13 children and CSF hypocretin assay in 6 cases.

Inclusion criteria: Children less than 18 years satisfying the diagnostic criteria proposed by the International Classification of Sleep Disorders for narcolepsy with cataplexy (narcolepsy type 1) and narcolepsy without cataplexy (narcolepsy type 2) were taken.

Exclusion criteria: Children with day time somnolence due to causes other than narcolepsy were excluded (Hypothyroidism, Obstructive sleep apnea, systemic diseases).

Results/Conclusion: Nineteen children (14 males and 5 females) were there in cohort. All 19 children had excessive day time somnolence. Nine children (47.3\%) had hypnagoic hallucinations, 7 (36.84\%) had cataplexy and none had sleep paralysis. Overnight PSG showed shortened sleep latency in 15 children, while normal sleep latency was seen in 4 children. MSLT showed shortened sleep latency and more than two SOREM in all children. HLA DQB $1 * 0602$ was positive in 13 patients and CSF hypocretin levels were measured in 6 cases, values were below $100 \mathrm{pcg} / \mathrm{ml}$. This is the largest case series of narcolepsy in children from India. Presentation of narcolepsy in children is similar to that reported in adults although typical cataplexy may not be present at the onset. Narcolepsy is a chronic disorder that remains under-diagnosed till adulthood with a significant impact on behavior and performance.
\end{abstract}

Keywords: Hyper somnolence, Sleep Attacks, Cataplexy, Narcolepsy, Hypnagogic-hallucination

Address for correspondence

\section{A. G. Roy}

Associate professor, Department of Neurology, AIMS, Ponnekkara lane, Kochi 682041, Kerala.

Email address: arungraceroy@aims.amrita.edu 


\section{Introduction}

$\mathrm{N}$

arcolepsy is a chronic disorder characterized by excessive daytime somnolence, sleep paralysis, hypnagogic or hypnopompic hallucinations when falling asleep or awakening respectively, cataplexy (narcolepsy tetrad), and disturbed night sleep (narcolepsy pentad). This disorder tends to have variable phenotype, although always associated with excessive daytime sleepiness. The pediatric narcolepsy population is increasingly being recognized. Symptoms of narcolepsy typically start in childhood and young adult. In this population, clinical picture can be more variable creating diagnostic challenges. This is especially in case of cataplexy which can be atypical affecting primarily the face causing grimaces or jaw opening with tongue thrusting a symptom known as "cataplectic faces". In this study, we are describing the epidemiology, clinical features, and investigations of 19 children with the diagnosis of narcolepsy.

\section{Methods}

Inclusion criteria: Children less than 18 years satisfying the diagnostic criteria proposed by the International Classification of Sleep Disorders for narcolepsy with cataplexy (Narcolepsy type 1) and narcolepsy without cataplexy (Narcolepsy type 2) were taken ${ }^{2}$. Sleep latency less than $8 \mathrm{~min}$ was taken as the minimum time for shortened sleep latency.

Exclusion criteria: Children with day time somnolence due to causes other than narcolepsy were excluded (Hypothyroidism, Obstructive sleep apnea, systemic diseases).

This is a descriptive retrospective study reporting on the clinical and biological features of children diagnosed with narcolepsy in the sleep center during period 2008-2011. Children (<18years) referred for symptoms of excessive day time somnolence were given a questionnaire that included questions on excessive day time somnolence, cataplexy, sleep paralysis, hallucinations, and behavioral disturbances. Detailed medical, drug, and family history were taken. Neurological examination and body mass index measurements were done in all children. All children underwent overnight polysomnography (PSG) followed by multiple sleep latency tests (MSLT). Routine blood investigations including thyroid function were done in all patients. HLA typing and CSF hypocretin analysis were done after informed consent. CSF hypocretin analysis was done in Stanford sleep center (Values $>200 \mathrm{pg} / \mathrm{ml}$ were considered normal and values $<110 \mathrm{pg} / \mathrm{ml}$ were considered diagnostic of narcolepsy). HLA typing was done in Stanford sleep center ( 6 children) and Shri Ganga Ram hospital (7 children).

\section{Results and Discussion}

A total of 19 children (14 males and 5 females) were there in this cohort. Children were divided into two groups pre-pubertal $(<12 y, 73.6 \%)$ and post pubertal $(>12 y, 26.4 \%)$ age groups. Average age of onset of disease was 9.3 years in males and 8.8 years in females. Age wise distribution is shown in Table-1. A Positive family history of excessive day time somnolence was seen in 5 patients in first degree relatives. The classical tetrad of narcolepsy was not seen in any children. All 19 children had excessive day time somnolence. Nine children (47.3\%) had hypnagoic hallucinations, 7 (36.84\%) had cataplexy (narcolepsy type 1) and none had sleep paralysis. In all cases excessive day time somnolence (EDS) was severe enough that it was reported to parents by school teachers. EDS occurred irrespective of the time of the day. Interestingly, while pre-pubertal age children did not realize they were excessively somnolent, but post pubertal age children were aware of the symptom but did not consider it to be abnormal. All children felt refreshed after short naps. As children were unable to take naps during school hours, $75 \%$ children reported feeling tired or dull during school.

Hypnagoic hallucinations described as "fearful visual dreams" when half awake were seen in 9 children. These mostly occur during night sleep but 3 of 19 subjects reported these experiences during day time naps. No auditory or tactile experiences were noted. Seven children had cataplexy. Localized cataplexy with jaw drop was seen in 6 children and was noticed by parents. Buckling of the knee associated with falls was seen in 1 child. Precipitating factor included laughter and anger. Behavioral disturbances (temper tantrums) were reported in 5 children and hyperactivity in 4 children. Automatic behavior and sleep paralysis were not reported. Frequent arousals from nocturnal sleep were reported by parents in 8 children. Excess appetite and weight gain were seen in 11 children.

Investigations: PSG followed by MSLT was performed 
in all 19 children. All children had shortened sleep latency in PSG, and 10 had a high arousal index. All children had a positive MSLT characterized by more than 2 SOREM and a mean sleep latency of less than $8 \mathrm{~min}$. The average mean sleep latency was 4 min with 6 patients having mean sleep latency below $1 \mathrm{~min}$. Average REM latency was $7.01 \mathrm{~min}$. HLA DQB $1^{*} 0602$ was positive in all 13 patients in whom it was tested. CSF hypocretin levels done in 6 cases, showed values below $100 \mathrm{pcg} / \mathrm{ml}$ indicative of hypocretin deficiency (5 with undetectable level). Neurological examination and thyroid function were normal in all children and neuroimaging was not performed.

Narcolepsy is a well defined disease entity in adult population, but reports are fewer in the pediatric age group. Here, the demographic and clinical features of 19 children with a diagnosis of narcolepsy are described. We found that most children in our study were prepubertal with the average age of 9.2 years in males and 8.8 years in females. Majority of the cases had onset of the symptoms between 7 and 9 years similar to other study of 97 pediatric cases $^{3}$. Retrospective studies in adults have shown that half of the patients reported onset in childhood ${ }^{4}$. The youngest age group in this series was 4 year old boy. Five children had family history of excessive somnolence in family members but as these have not been evaluated for narcolepsy it is difficult to conclude if it could be narcolepsy. Previous studies have reported a risk for narcolepsy cataplexy of $1 \%$ for first degree relatives (20 - to 40 -fold increase in the risk of having this disease) and up to $4 \%$ for unexplained day time sleepiness 5 .

EDS was seen in all 19 children. This symptom is prerequisite for diagnosis of narcolepsy with or without cataplexy according to International Classification of Sleep Disorders for narcolepsy with cataplexy and narcolepsy without cataplexy. It has to be present for minimum 3 months. Duration of EDS was more than 6 months in all children in our series. This was mainly noted by school teachers as children appearing dull and dozing off during school hours. Interestingly, most parents did not consider EDS as pathological in their children interpreting it as laziness or as part of a physiological evolution. The majority of children felt they were tired through out day and less active compared to their peers. This has been noticed in previous studies also where EDS was missed as symptom ${ }^{1,6}$. In healthy children, physiological and scheduled daytime napping rapidly declines between the age of 18 months and 5 years ${ }^{6}$. This was the first symptom in all children and causing most distress to the patient ${ }^{7}$.

Cataplectic attacks usually provoked by strong emotion (most often by laughter) are reported in $80.5 \%$ of idiopathic cases ${ }^{8}$. In cataplexy loss of muscle tone is bilateral, and in the most severe instance can results in a fall. Consciousness is usually unaffected and the patient is aware but paralyzed. Most cataplexy attacks subside within a minute and there is no postictal confusion. Cataplexy generally occurs in $60-90 \%$ of patients with narcolepsy and its onset follows the onset of sleepiness by several months to years. In our study, cataplexy was found in only 7 children (36\% cases). Jaw drop was seen in 6 children and one had knee buckling and falls. These were provoked by an emotional outburst like laughing and occasionally crying. Each attack lasted for 10-15 s and occurred multiple times a day. The child with cataleptic falls was misdiagnosed as seizure and was on antiepileptic drugs. The onset of cataplexy was about 23 months after the onset of EDS. The low incidence of cataplexy in our study group highlights the fact that typical cataplexy may be a later manifestation of narcolepsy compared to EDS. In recent reports, cataplexy has been reported as a typical affecting face causing grimaces or jaw opening with tongue thrusting, a symptom known as cataplectic faces ${ }^{1}$.

In this study, hypnogogic hallucinations were present in $47 \%$ cases. Hallucinations were in the visual domain in these patients and were formed images like colored circles and animals. Hallucinations occurred mostly during the nocturnal sleep and occasionally during day time naps. In prior reports, hypnagogic and hypnopompic hallucinations occurred in approximately $60 \%$ of the patients which is similar to this study. These can be visual, auditory, or out-of-body experiences. In children the hallucinations are usually of simple forms ${ }^{9,10}$. As previously noted, children behavioral disturbances emerged in some of this cases with onset of disease. In this series, 5 children had temper tantrums and hyperactivity was present in 4 children. Behavior changes such as sorrowfulness, emotional lability, irritability or aggressiveness have been reported ${ }^{11}$. A multicentre study with children suffering from narcolepsy showed higher rates of behavioral problems and depression when compared to controls ${ }^{12}$.

Obesity ( $>$ 95th centile of the expected BMI for age) was seen in 11 children in this series. The onset of obesity 
was within one month of EDS in all the children. Obesity is a common finding in narcolepsy ${ }^{13}$. The weight gain occurs despite of lower caloric intake and without clear association to inactivity or drugs ${ }^{14-16}$. In children, the weight gain occurs suddenly after the onset of other symptoms of narcolepsy ${ }^{17}$ and may be related to abnormal hypocretin and leptin levels and effects on energy metabolism ${ }^{8,19}$. Another study devoted to eating disorders and metabolism in narcoleptic patients revealed that narcoleptic patients have a lower basal metabolism than the controls ${ }^{20}$.

An overnight PSG followed by next day MSLT is the investigation of choice for diagnosing narcolepsy. Studies suggest that by the age of 8 years children typically have adult features on the MSLT ${ }^{3,21}$. In toddlers and young preschool children, continuous daytime records using the ambulatory monitoring technique can be a useful alternative diagnostic method although this is not well validated either ${ }^{11}$. Serial sleep studies may be required in children to establish a definitive diagnosis ${ }^{22}$. Narcoleptics with cataplexy had a higher number of SOREM and much shorter mean sleep latency in MSLT than narcoleptics without cataplexy but this difference was not seen in this series ${ }^{23}$. In this series, overnight PSG showed shortened sleep latency in 15 children, while normal sleep latency was seen in 4 children. MSLT showed shortened sleep latency and more than two SOREM in all children. This stressed the need for MSLT since latency can be normal in overnight PSG. Frequent arousals were also seen in 10 children in nocturnal PSG. Overnight PSG is useful in excluding other causes of disturbed sleep causing day time somnolence like parasominas, obstructive sleep apnea.

More than $90 \%$ of Caucasian patients with a sporadic narcolepsy cataplexy share a specific HLA DR2/15 allele, HLADQB ${ }^{*} 0602^{11}$. Recent studies have shown that HLADQA $1 * 0102$ and DQB $1 * 0602$ alleles found in $25 \%$ of general population are primary susceptibility factors for narcolepsy $y^{24}$. Its sensitivity is highest in patients with narcolepsy and cataplexy, a group in which additional diagnostic test is seldom necessary ${ }^{1}$. In this series, HLADQB $1{ }^{*} 0602$ was positive in all patients who are diagnosed irrespective of whether they had cataplexy or not. CSF hypocretin is another biomarker of narcolepsy and was low in 6 patients who are diagnosed. Kanbayashi et al. ${ }^{25}$ showed that the CSF level of Hcrt-1 remains stable from early infancy up to old age and undetectable level of Hcrt-1 in CSF is a very valuable diagnostic marker in children. In pre-pubertal children, undetectable Hcrt-1 levels in CSF can be seen even if PSG is normal ${ }^{26}$. CSF Hcrt-1 level below $110 \mathrm{pg} / \mathrm{mL}$ is highly indicative of narcolepsy, though a higher level does not exclude the diagnosis ${ }^{1}$.

Brain imaging is indicated when a symptomatic cause for narcolepsy is suspected. Children tend to have symptomatic causes more than adult patients. Symptomatic narcolepsy should be suspected in cases where narcolepsy has onset in young children, cataplectic attacks are abnormally frequent, and when PSG evidence of classical narcolepsy is absent or where human leukocyte antigen typing for DR2 is negative ${ }^{8}$. In this series, brain imaging was not performed as cases were all confirmed typical. The clinical features and investigations are similar to previous reported case series in children of Chinese and Caucasian ethnicity. Unlike other reports, clustering of cases or association with vaccination or infections were not found.

Treatment is life long and depends on symptoms of patients and includes drug therapy and life style modification. The most common drugs used in children are modafinil, atomoxetine, and methylphenidate for $\mathrm{EDS}^{1,11,27}$. For cataplexy, venlafaxin, clomipramine, and fluoxetine are most often prescribed and dose should be based on body weight ${ }^{1,11,27}$. The role of Sodium oxybate appears to be an effective and well-tolerated treatment for all the key symptoms of narcolepsy and large studies are lacking in children but case reports have shown benefit in children.

\section{Conclusion}

This is the largest case series of narcolepsy in children from India. Presentation of narcolepsy in children is similar to that reported in adults although typical cataplexy may not be present at the onset. Narcolepsy is a chronic disorder that remains under diagnosed till adulthood with a significant impact on behavior and performance and has been noted in previous studies ${ }^{28,29}$. Physicians should ask about sleep history in all children and if there is history of excessive day time somnolence, the child should be definitely be evaluated for narcolepsy. PSG followed by a day time MSLT is diagnostic tool of choice for narcolepsy but HLA typing and CSF hypocretin measurement are useful if further confirmation is needed.

Indian Journal of Sleep Medicine (IJSM), Vol. 10, No. 2, 2015 
Table 1: Age and sex distribution of the study group.

\begin{tabular}{|l|l|l|}
\hline Age Group & Male & Female \\
\hline$<5$ years & 1 & 0 \\
\hline $5-10$ years & 11 & 3 \\
\hline $12-18$ years & 2 & 2 \\
\hline Total & $14(73.68 \%)$ & $5(26.31 \%)$ \\
\hline
\end{tabular}

\section{References}

1. Paul C. Peterson, Aatif M. Husain: Pediatric narcolepsy. Brain \& Development 30 2008; 609-23.

2. AASM. International classification of sleep disorders. 2nd ed.Westchester IL: American Academy of Sleep Medicine; 2005.

3. Challamel MJ, Mazzola ME, Nevsmalova S, Cannard C, Louis J, Revol MI. Narcolepsy in children. Sleep 1994;17(Suppl. 8):S17-20.

4. McKenna C, McNicholas F. Childhood onset narcolepsy. A case report. Eur Child adolesc Psychiatry 2003; 12:43-7.

5. Ohayon MM, Ferini-Strambi L, Plazzi G, Smirne S, Castronovo V. Frequency of narcolepsy symptoms and other sleep disorders in narcoleptic patients and their first-degree relatives. J Sleep Res 2005;14:437-45.

6. Stores G. The protean manifestations of childhood narcolepsy and their misinterpretation. Dev Med Child Neurol 2006;48(4):307-10.

7. Adi Aran, Mali Einen, Ling Lin, Guiseppe Plazzi, Seiji Nishino. Clinical and therapeutic aspects of childhood narcolepsy-cataplexy: a retrospective study of 51 children. Sleep $2010 ; 33(11)$ : 1457-64.

8. Challamel MJ, Mazzola ME, Nevsimalova S, Cannard C, Louis J, Revol M. Narcolepsy in children. Sleep. 1994;17(8 Suppl):S17-20.

9. Overeem S, Mignot E, van Dijk JG, Lammers GJ. Narcolepsy: Clinical features, new pathophysiologic insights and future perspectives. J Clin Neurophysiol 2001; 18(2):78-105.

10. Thorpy M. Current concepts in the etiology, diagnosis and treatment of narcolepsy. Sleep Med 2001;2(1):5-17.

11. Sona Nevsimalova. Narcolepsy in childhood. Sleep Medicine Reviews 2009;13: 169-80.

12. Stores G, Montgomery P, Wiggs L. The psychological problems of children with narcolepsy and those with excessive daytime sleepiness of uncertain origin. Pediatrics 2006; 118:111623.

13. Okun ML, Lin L, Pelin Z, Hong S, Mignot E. Clinical aspects of narcolepsy-cataplexy across ethnic groups. Sleep 2002; 25(1):27-35.
14. KokSW, OvereemS, Visscher TL, Lammers GJ, Seidell JC, $\mathrm{Pijl} \mathrm{H}$, Meinders A E . Hypocretin deficiency in narcoleptic humans is associated with abdominal obesity. Obes Res 2003; $11(9): 1147-54$.

15. Lammers GJ, Pijl H, lestra J, Langius JA, Buunk G, Meinders AE. Spontaneous food choice in narcolepsy. Sleep 1996; 19(1):75-6.

16. Schuld A, Beitinger PA, Dalal M, Geller F, Wetter TC, AlbertED, et al. Increased body mass index (BMI) in male narcoleptic patients, but not in HLA-DR2-positive healthy male volunteers. Sleep Med 2002; 3(4):335-9.

17. Kotagal S, Krahn LE, Slocumb N. A putative link between childhood narcolepsy and obesity. Sleep Med $2004 ; 5(2): 147-50$.

18. Crocker A, Espana RA, Papadopoulou M, Saper CB, Faraco $\mathrm{J}$,Sakurai T, et al. Concomitant loss of dynorphin, NARP, and orexin in narcolepsy. Neurology 2005;65(8):1 184-8.

19. Nishino S, Ripley B, Overeem S, Nevsimalova S, Lammers GJ, Vankova J, et al. Low cerebrospinal ûuid hypocretin (orexin) and altered energy homeostasis in human narcolepsy. Ann Neurol 2001; 50:381-8.

20. Chabas D, Foulon C, Gonzales J, Nasr M, Caen OL, Willer $\mathrm{JC}$, et al. Eating disorder and metabolism in narco leptic patients. Sleep 2007;30:1267-73.

21. Guilleminault C, Pelayo R. Narcolepsy in prepubertal children. Ann Neurol 1998;43(1):135-42.

22. Kotagal S, Goulding PM. The laboratory assessment of daytime sleepiness in childhood. J Clin Neurophysiol $1996 ; 13: 208-18$.

23. Huang YS, Tafti M, Guilleminault C. Daytime sleepiness with and without cataplexy in ChineseeTaiwanese patients. Sleep Med 2006;7:454-7.

24. Kadotani $\mathbf{H}$, Faraco J, Mignot E. Genetic studies in the sleep disorder narcolepsy. Genome Res September 5, 2007;8:427-34.

25. Kanbayashi T, Yano T, Ishiguro H, Kawanishi K, Chiba S, Aizawa R, et al. Hypocretin (orexin) levels in human lumbarCSF in different age groups: infants to elderly persons. Sleep 2002;25:337-9.

26. Kubota H, Kanbayashi T, Tanabe $Y$, Ito $M$, Takanashi J, Kohno Y, et al. Decreased cerebrospinal ûvid hypocretin-1 levels near the onset of narcolepsy in 2 pre-pubertal children. Sleep 2003; 26:55-57.

27. Greenhill LL, Pliszka S, Dulcan MK, Bernet W, Arnold $\mathrm{V}$,Beitchman J, et al. Practice parameter for the use of stimulantmedications in the treatment of children, adolescents, and adults. J Am Acad Child Adolesc Psychiatry 2002;41(Suppl. 2):26-49.

28. Merrill S.Wise, Jennifer Lynch. Narcolepsy in children. Seminars in Pediatric Neurology 2001; Vol 8, (4) :198206

29. Young D, Zorick F, Wittig R, Roehrs T, Roth T. Narcolepsy in a pediatric population. Am J Dis Child. 1988 Feb; $142(2): 210-3$ 